\title{
Maternal and Fetal Outcomes in Systemic Lupus Erythematosus Pregnancies
}

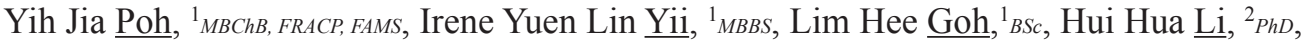
Liying Yang, ${ }^{3}$ MBBS, MMed (O\&G), FAMS, Hak Koon Tan, ${ }^{3} M B B S$, FRCOG (UK), FAMS, Julian Thumboo, ${ }^{1} M B B S$, FRCP (Edin), FAMS, Lay Kok Tan, ${ }^{3} M B B S$, FRCOG (UK), FAMS

\begin{abstract}
Introduction: To describe the maternal and fetal outcomes in systemic lupus erythematosus (SLE) pregnancies followed-up in a single tertiary referral centre.

Methods: We performed a retrospective cohort study of 75 SLE pregnancies who were followed up in Singapore General Hospital over a 16-year period from 2000 to 2016. Adverse fetal and maternal outcomes including preterm delivery, miscarriages, fetal growth restriction, congenital heart block, neonatal lupus, pre-eclampsia and SLE flares were obtained from the medical records.

Results: The mean age at conception was 32 years old (SD 3.8). The mean SLE disease duration was 5.9 years (SD 5.2). The majority (88\%) had quiescent SLE disease activity at baseline. Most pregnancies resulted in a live birth $(74.7 \%)$. The mean gestational age at birth was 37.4 weeks (SD 3.4). Adverse fetal outcomes occurred in $53.3 \%$. Preterm delivery $(33.9 \%)$, miscarriages $(20 \%)$ and fetal growth restriction $(17.3 \%)$ were the most frequent adverse fetal outcomes. There was 1 neonatal death and SLE flares occurred in a third (33\%). In the subgroup of SLE pregnancies with antiphospholipid syndrome, there were higher SLE flare rates $(40 \%)$ and adverse fetal outcomes occurred in 8 pregnancies ( $80 \%$ ). There were no predictive factors identified for all adverse fetal and maternal outcomes. In the subgroup analysis of preterm delivery, anti-Ro (SS-A) antibody positivity and hydroxychloroquine treatment were associated with a lower risk of preterm delivery.

Conclusion: Although the majority had quiescent SLE disease activity at baseline, SLE pregnancies were associated with high rates of adverse fetal and maternal outcomes.

Ann Acad Med Singap 2020;49:963-70

Keywords: Antiphospholipid syndrome, anti-La (SS-B) antibody, anti-Ro (SS-A) antibody, lupus nephritis
\end{abstract}

\section{Introduction}

Systemic lupus erythematosus (SLE) is a systemic autoimmune disease that primarily affects women of childbearing age. ${ }^{1}$ In addition, SLE women have a normal fertility rate and therefore pregnancy is an important aspect to discuss and manage in these patients. $^{2,3}$ Although pregnancy outcomes in SLE women have improved over time, SLE pregnancies remain high risk. SLE pregnancies are associated with an increased risk of adverse maternal and fetal outcomes including preterm delivery, fetal growth restriction (FGR), pre-eclampsia, fetal loss, hypertensive disorders of pregnancy and maternal morbidity and mortality. ${ }^{4-6}$

\footnotetext{
${ }^{1}$ Department of Rheumatology and Immunology, Singapore General Hospital, Singapore

${ }^{2}$ Health Services Research Unit, Singapore General Hospital, Singapore

${ }^{3}$ Department of Obstetrics and Gynaecology, Singapore General Hospital, Singapore

Address for Correspondence: Dr Yih Jia Poh, Department of Rheumatology and Immunology, Academia Building, Level 4, 20 College Road, Singapore General Hospital, Singapore 169856.

Email: poh.yih.jia@singhealth.com.sg
} 
There have also been conflicting results from previous studies on the effect of pregnancy on disease activity in SLE, and variable flare rates between 19 and 68\% have been reported..$^{7-10}$ Numerous predictors of flares have been reported including SLE disease activity 6-12 months prior to conception and cessation of hydroxychloroquine. ${ }^{11,12}$ Limited data exist on pregnancy outcome in Southeast-Asian SLE patients. This study aims to describe the maternal and fetal outcomes in SLE pregnancies followed up in a tertiary referral centre in Singapore.

\section{Methods}

We performed a retrospective cohort study of pregnant patients with SLE who were followed up in Singapore General Hospital, a tertiary referral centre in Singapore, over a 16-year period from 2000 to 2016. All pregnant women who underwent antenatal follow-up in the high risk pregnancy clinic and met the 1997 American College of Rheumatology (ACR) classification criteria for $\mathrm{SLE}^{13}$ were included in the study. Each pregnancy was counted as a separate observation.

Clinical characteristics recorded included maternal demographic profile, prior obstetrics history, co-morbidities, medications, prior immunosuppressant therapy, presence of antiphospholipid syndrome (APS), SLE clinical manifestations, disease activity and disease duration of SLE prior to conception. SLE disease activity was evaluated according to the Safety of Estrogens in Lupus Erythematosus National Assessment (SELENA) version of the Systemic Lupus Erythematosus Disease Activity Index (SLEDAI) scale. ${ }^{14}$ Patients were categorised into inactive SLE (SELENA-SLEDAI score $<6$ ) or active SLE (SELENA-SLEDAI score $\geq 6$ ) according to their SELENA-SLEDAI scores. APS was defined according to the Sapporo criteria. ${ }^{15}$ Immunologic characteristics of patients were recorded and included antiphospholipid antibodies (aPL), anti-Ro (SS-A) and anti-La (SS-B) antibodies.

Maternal outcomes reviewed included SLE disease activity, SLE flares, pre-eclampsia, eclampsia, placenta praevia, maternal death, gestational diabetes, venous thromboembolic events and mode of delivery. Adverse maternal outcome was defined as pre-eclampsia, eclampsia, placenta praevia, gestational diabetes, maternal death, venous thromboembolic events or SLE flares.

Fetal outcomes evaluated were live births, Apgar scores at 1 minute and 5 minutes, birth weight, gestational age, fetal loss which included termination of pregnancy, miscarriages and intra-uterine fetal death and presence of fetal growth restriction (FGR). FGR was defined as a fetal weight that was below the 10th percentile for gestational age. Live births were categorised into preterm and term. Preterm delivery was defined as delivery before 37 weeks of gestation and term birth as delivery $\geq 37$ weeks of gestation. Miscarriages were categorised into early miscarriage defined as fetal loss prior to 10 weeks' gestation and late miscarriage defined as fetal loss $\geq 10$ weeks' gestation. Intrauterine fetal death was defined as a baby delivered with no signs of life known to have died after 24 weeks gestation. ${ }^{16}$ An Apgar score $>7$ was defined as normal whereas a score $<7$ was considered to indicate moderate or severe hypoxia. ${ }^{17}$ Adverse fetal outcome was defined as miscarriages, intrauterine fetal death, FGR, preterm delivery, fetal abnormality, congenital heart block or neonatal lupus.

\section{Statistical analysis}

Descriptive statistics were presented as frequency, mean and standard deviation (SD) measures. Categorical data and mean, together with SD were reported for continuous data. Mixed model was performed to evaluate the effects of potential factors on preterm delivery and miscarriages. All analysis was performed with R3.6.2 (www.r-project.org). A statistical significance level of 5\% $(P<0.05)$ was utilised.

This study was approved by the SingHealth Centralised Institutional Review Board (CIRB 2014/2056) with exemption of patient consent.

\section{Results}

\section{Patient characteristics}

There was a total of 75 pregnancies in 45 SLE women during the observation period from 2000 to 2016. All the patients fulfilled the 1997 ACR criteria for SLE diagnosis. ${ }^{13}$ In our multi-ethnic cohort, $65.3 \%$ were Chinese, 22.7\% Malay and 6.7\% Indian. The mean age at conception was 32 years old (SD 3.8). There were 2 in-vitro fertilisation pregnancies. The mean SLE disease duration prior to conception was 5.9 years (SD 5.2). At baseline, the majority (88\%) had quiescent SLE disease activity. Patient characteristics are summarised in Table 1.

The most frequent pregestational SLE clinical manifestations were haematological (73.3\%), arthritis (70.7\%), renal (57.4\%), mucocutaneous (25.3\%), serositis $(20 \%)$ and neurological $(10.7 \%)$. In the 43 pregnancies with renal involvement, there were 30 with a prior renal biopsy. More than half $(56.7 \%)$ had Class IV lupus nephritis, 23.3\% Class V lupus nephritis and 16.7\% with Class III lupus nephritis on renal biopsy.

There were 10 pregnancies (13.3\%) with secondary APS and 9 of these were obstetric APS. There was 1 
Table 1. Baseline demographics and clinical characteristics of pregnant SLE women

\begin{tabular}{|c|c|}
\hline $\begin{array}{l}\text { Age at conception (years) } \\
\text { Mean } \pm \text { SD }\end{array}$ & $32.0 \pm 3.8$ \\
\hline $\begin{array}{l}\text { Age at diagnosis of SLE (years) } \\
\text { Mean } \pm \text { SD }\end{array}$ & $26.0 \pm 5.5$ \\
\hline $\begin{array}{l}\text { SLE disease duration (years) } \\
\text { Mean } \pm \text { SD }\end{array}$ & $5.9 \pm 5.2$ \\
\hline $\begin{array}{l}\text { Blood pressure at booking visit }(\mathrm{mmHg}) \\
\text { Mean } \pm \text { SD }\end{array}$ & $104.4 \pm 13.4$ \\
\hline $\begin{array}{l}\text { Ethnicity, } \boldsymbol{n}(\%) \\
\text { Chinese } \\
\text { Malay } \\
\text { Indian } \\
\text { Others }\end{array}$ & $\begin{array}{l}49(65.3) \\
17(22.7) \\
5(6.7) \\
4(5.3)\end{array}$ \\
\hline Smoking, n (\%) & $8(10.7)$ \\
\hline $\begin{array}{l}\text { Previous deliveries, } \boldsymbol{n}(\%) \\
\text { None } \\
\text { One or more }\end{array}$ & $\begin{array}{l}33(44.0) \\
42(56.0)\end{array}$ \\
\hline $\begin{array}{l}\text { Previous SLE specific treatment, } \boldsymbol{n}(\%) \\
\text { Prednisone } \\
\text { Hydroxychloroquine } \\
\text { Chloroquine } \\
\text { Azathioprine } \\
\text { Mycophenolate mofetil } \\
\text { Cyclosporin } \\
\text { Methotrexate } \\
\text { Cyclophosphamide } \\
\text { Rituximab }\end{array}$ & $\begin{array}{l}71(94.7) \\
48(64.0) \\
9(12.0) \\
28(37.3) \\
21(28.0) \\
6(8.0) \\
1(1.3) \\
14(18.7) \\
3(4.0)\end{array}$ \\
\hline $\begin{array}{l}\text { Co-morbidities, } \boldsymbol{n} \text { (\%) } \\
\text { Hypertension } \\
\text { Hyperlipidaemia } \\
\text { Diabetes mellitus } \\
\text { Thyroid disease }\end{array}$ & $\begin{array}{l}1(1.3) \\
1(1.3) \\
1(1.3) \\
2(2.7)\end{array}$ \\
\hline $\begin{array}{l}\text { SLE clinical manifestations, } \boldsymbol{n}(\%) \\
\text { Haematological } \\
\text { Arthritis } \\
\text { Renal } \\
\text { Muco-cutaneous } \\
\text { Serositis } \\
\text { Neurological }\end{array}$ & $\begin{array}{l}55(73.3) \\
53(70.7) \\
43(57.4) \\
19(25.3) \\
15(20.0) \\
8(10.7)\end{array}$ \\
\hline $\begin{array}{l}\text { Concomitant rheumatic disease, } \boldsymbol{n}(\%) \\
\text { Sjogren's Syndrome } \\
\text { Antiphospholipid Syndrome }\end{array}$ & $\begin{array}{l}26(34.7) \\
10(13.3)\end{array}$ \\
\hline $\begin{array}{l}\text { Immunological characteristics, } \boldsymbol{n}(\%) \\
\text { anti-Ro (SS-A) } \\
\text { anti-La (SS-B) } \\
\text { anti-cardiolipin IgG } \\
\text { anti-cardiolipin IgM } \\
\text { Lupus anticoagulant }\end{array}$ & $\begin{array}{l}33(44.0) \\
7(9.3) \\
17(22.7) \\
14(18.7) \\
10(13.3)\end{array}$ \\
\hline $\begin{array}{l}\text { Current Medications, } \boldsymbol{n} \text { (\%) } \\
\text { Prednisone } \\
\text { Hydroxychloroquine } \\
\text { Chloroquine } \\
\text { Azathioprine } \\
\text { Cyclosporine } \\
\text { Aspirin } \\
\text { Low molecular weight heparin }\end{array}$ & $\begin{array}{l}57(76.0) \\
40(53.3) \\
8(10.7) \\
20(26.7) \\
1(1.3) \\
25(33.3) \\
14(18.7)\end{array}$ \\
\hline
\end{tabular}

Table 1. Baseline demographics and clinical characteristics of pregnant SLE women (Cont'd)

\begin{tabular}{ll}
\hline Prior fetal outcome, $\boldsymbol{n}$ (\%) & \\
Total pregnancies & 103 \\
Live birth $^{\mathrm{a}}$ & $63(61.2)$ \\
Term delivery & $25(39.7)$ \\
Pre-term delivery & $13(30.2)$ \\
Miscarriage & $27(26.2)$ \\
$\quad$ Early miscarriage (<10 weeks' gestation) & $16(59.3)$ \\
Late miscarriage ( ${ }^{3} 10$ weeks' gestation) & $3(11.1)$ \\
Terminations & $13(12.6)$ \\
FGR & $1(0.9)$ \\
Neonatal death & $2(1.9)$ \\
Prior maternal outcome, $\boldsymbol{n}$ (\%) & \\
Total pregnancies & 103 \\
Pre-eclampsia & $2(1.9)$ \\
Eclampsia & $0(0)$ \\
Placenta praevia & $1(0.9)$ \\
Maternal death & $0(0)$ \\
SLE disease activity at baseline & \\
SELENA-SLEDAI, mean (range) & $0.43(0-8)$ \\
\hline
\end{tabular}

SLE: systemic lupus erythematosus; FGR: fetal growth restriction; SELENA-SLEDAI: Safety of Estrogens in Lupus Erythematosus National Assessment (SELENA) version of the Systemic Lupus Erythematosus Disease Activity Index (SLEDAI) scale ${ }^{\mathrm{a}}$ Missing $=19,{ }^{\mathrm{b}}$ Missing $=8$

patient with the clinical phenotype of thrombotic APS and had a prior pulmonary embolism. There were 5 patients with concomitant co-morbidities including thyroid disease $(n=2)$, hypertension $(n=1)$, diabetes mellitus $(n=1)$ and hyperlipidaemia $(n=1)$. A third of the cohort $(\mathrm{n}=26)$ had Sjogren's syndrome.

There were 33 (44\%) who were primigravida in our cohort. In the multiparous SLE women, there were a total of 103 previous pregnancies and the live birth rate was $61.2 \%$. There were a third (30.2\%) with preterm deliveries. Miscarriages occurred in 27 pregnancies $(26.2 \%)$ and the majority of these $(59.3 \%)$ were early miscarriages. Preeclampsia occurred in 2 pregnancies $(1.9 \%)$.

\section{Medications}

Prior to pregnancy, the most frequent SLE-specific therapy prescribed were corticosteroid $(94.7 \%)$, antimalarial including hydroxychloroquine and chloroquine (76\%), and azathioprine (37.3\%). There were 14 pregnancies $(18.7 \%)$ with a history of cyclophosphamide therapy. During pregnancy, the most common SLE-specific therapy was similar with the majority receiving corticosteroids (76\%) and antimalarials (64\%). The mean dose of prednisone was $7.4 \mathrm{mg}$ daily. Low-dose aspirin and low-molecular weight heparin (LMWH) were administered in 25 pregnancies $(33.3 \%)$ and 14 pregnancies $(18.7 \%)$, respectively.

In the subgroup of SLE with secondary APS $(n=10)$, 4 were treated with therapeutic dose of LMWH 
$(1 \mathrm{mg} / \mathrm{kg}), 1$ with a combination of therapeutic LMWH and aspirin, 1 with prophylactic dose of LMWH (40mg daily), and 1 with a combination of prophylactic LMWH and aspirin.

\section{Immunological characteristics}

There were 33 pregnancies (44\%) with anti-Ro (SS-A) antibody positivity and 7 pregnancies $(9 \%)$ with antiLa (SS-B) antibody positivity. The prevalence of aPL positivity was $40 \%$, with anticardiolipin (aCL) antibody of immunoglobulin $\mathrm{G}$ ( $\mathrm{IgG})$ being the most common (22.7\%), followed by aCL antibody of immunoglobulin $\mathrm{M}(\mathrm{IgM})(18.7 \%)$ and lupus anticoagulant (13.3\%).

\section{Fetal outcome}

In our cohort, the live birth rate was $74.7 \%$. More than half of the deliveries $(57.1 \%)$ were via caesarean section. There were 37 pregnancies $(66.1 \%)$ with term deliveries. The mean gestational age at birth was 37.4 weeks (23-40.6 weeks, SD 3.4) and mean fetal weight was 2669.3g (585-3875g, SD 723.9). There were 3 neonates with Apgar score $<7$ at 1 minute and none with Apgar score $<7$ at 5 minutes. Pregnancy outcomes are summarised in Table 2.

Adverse fetal outcomes occurred in 40 pregnancies $(53.3 \%)$. Preterm delivery was the most common complication and occurred in 19 pregnancies (33.9\%) with 2 extremely preterm birth ( $<28$ weeks' gestation) and 2 very preterm birth ( 28 weeks to $<32$ weeks' gestation). There were 15 pregnancies with miscarriages and the majority $(13 / 15)$ were early pregnancy losses that occurred prior to 10 weeks' gestation. There were 13 pregnancies $(17.3 \%)$ with FGR. There was 1 neonatal death due to neonatal sepsis. There were no cases of congenital heart block or neonatal lupus in our cohort.

In the subgroup of SLE with secondary APS pregnancies, adverse fetal outcome occurred in 8 pregnancies $(80 \%)$. The live birth rate was $50 \%$ with 3 preterm deliveries. Miscarriages occurred in 4 pregnancies with 2 early miscarriages and 2 late miscarriages. FGR occurred in 2 pregnancies as shown in Table 2.

In SLE pregnancies with lupus nephritis, adverse fetal outcome occurred in 21 pregnancies $(48.8 \%)$. The live birth rate was $62.8 \%$ and the majority $(70.4 \%)$ were term deliveries. Miscarriages occurred in 10 pregnancies with $80 \%$ of these being early miscarriages (Table 2).

We evaluated the potential factors associated with preterm delivery, miscarriages, live births and all adverse fetal outcomes. Univariable mixed model analysis revealed that anti-Ro (SS-A) antibody positivity (OR 0.41, CI 0.416-0.420) and hydroxychloroquine treatment (OR 0, CI 0-0.054) were associated with a lower risk of preterm delivery. Results are summarised in Table 3. In the subgroup of anti-Ro (SS-A) antibody positive pregnancies, more than half $(60.6 \%)$ were treated with antimalarials (hydroxychloroquine or chloroquine). Antimalarial prescription was similar in the subgroup without anti-Ro (SS-A) or anti-La (SS-B) antibody positivity; with more than half (66.7\%) who received antimalarial. There were $42.3 \%$ with lupus nephritis, $19.2 \%$ with APS and lupus nephritis and $3.8 \%$ with APS in the subgroup of anti-Ro (SS-A) antibody positive pregnancies. The majority (93.9\%) had quiescent SLE disease activity at baseline that was comparable to the whole cohort. There was a trend towards increased risk of preterm delivery in Malays (OR 4.21, 95\% CI 0.906-19.580) although this was not statistically significant. In our analysis, there were no predictive factors associated with live births, miscarriages or combined adverse fetal outcomes.

\section{Maternal outcome}

There were no predictive factors that were associated with all adverse maternal outcomes. SLE flares occurred in a third (25/75) of our cohort. The most frequent organ involvement was haematological (44\%), renal (40\%), mucocutaneous (28\%) and musculoskeletal $(16 \%)$. There were 3 patients with no prior renal involvement who experienced a first renal flare in pregnancy. There were 5 pregnancies with a post-partum SLE flare. In the post-partum period, the most common organ involvement was musculoskeletal $(60 \%)$.

Gestational diabetes occurred in 4 pregnancies $(5.3 \%)$ and pre-eclampsia in 3 pregnancies $(4 \%)$. There was 1 pregnancy with post-partum haemorrhage requiring blood transfusion and subsequent hysterectomy.

In the SLE with APS pregnancies, SLE flares occurred in 4 pregnancies (40\%) and half of these were renal flares. There was 1 obstetric APS patient who developed deep venous thrombosis 4 weeks post-partum while receiving prophylactic dose of LMWH.

In the subgroup of SLE with lupus nephritis, SLE flares occurred in 14 pregnancies $(32.6 \%)$. The most common organ involvement was renal $(50 \%)$, followed by mucocutaneous (35.7\%) and haematological (35.7\%).

\section{Discussion}

In our cohort, the live birth rate was $74.7 \%$, which is comparable to other cohorts. ${ }^{18-20}$ Although a majority of patients $(88 \%)$ had quiescent SLE disease at conception, adverse fetal outcome as defined by miscarriages, 
Table 2. Maternal and fetal outcomes in the overall cohort, SLE with secondary APS and SLE with lupus nephritis

\begin{tabular}{|c|c|c|c|}
\hline & All SLE pregnancies & $\begin{array}{c}\text { SLE with secondary } \\
\text { APS }\end{array}$ & $\begin{array}{c}\text { SLE with lupus } \\
\text { nephritis }\end{array}$ \\
\hline Number of pregnancies, $n$ & 75 & 10 & 43 \\
\hline Live birth, n (\%) & $56(74.7)$ & $5(50)$ & $27(62.8)$ \\
\hline Term delivery & $37(66.1)$ & $2(40)$ & $19(70.4)$ \\
\hline Pre-term delivery & $19(33.9)$ & $3(60)$ & $8(29.6)$ \\
\hline \multicolumn{4}{|l|}{ Mode of delivery, $n(\%)$} \\
\hline Instrumental delivery & $4(7.1)$ & $0(0)$ & $2(7.4)$ \\
\hline Caesarean section & $32(57.1)$ & $4(80)$ & $12(44.4)$ \\
\hline Gestational age (weeks), mean $\pm \mathrm{SD}$ & $37.4 \pm 3.4$ & $32.26 \pm 6.3$ & $36.2 \pm 3.9$ \\
\hline Fetal weight (grams), mean \pm SD & $2669.3 \pm 723.9 \mathrm{u}^{\mathrm{a}}$ & $1485.3 \pm 1042.1$ & $2559.8 \pm 806.7^{\mathrm{d}}$ \\
\hline \multicolumn{4}{|l|}{ Apgar score, $n(\%)$} \\
\hline Apgar $<7$ at 1 minute & $3(5.4)^{b}$ & $0(0)^{\mathrm{c}}$ & $1(3.7)$ \\
\hline Apgar $<7$ at 5 minutes & $0(0)^{\mathrm{b}}$ & $0(0)^{\mathrm{c}}$ & $0(0)$ \\
\hline Early miscarriage (<10 weeks) & $13(86.7)$ & $2(50)$ & $8(80.0)$ \\
\hline Late miscarriage ( $\left({ }^{3} 10\right.$ weeks) & $2(13.3)$ & $2(50)$ & $2(20.0)$ \\
\hline Termination, $\mathrm{n}(\%)$ & $4(5.3)$ & $1(10)$ & $4(9.3)$ \\
\hline Neonatal death, n (\%) & $1(1.3)$ & $0(0)$ & $1(2.3)$ \\
\hline FGR, n (\%) & $13(17.3)$ & $2(20)$ & $3(6.9)$ \\
\hline Fetal abnormality, n (\%) & $0(0)$ & $0(0)$ & $0(0)$ \\
\hline Congenital heart block, n (\%) & $0(0)$ & $0(0)$ & $0(0)$ \\
\hline Neonatal lupus, n (\%) & $0(0)$ & $0(0)$ & $0(0)$ \\
\hline \multicolumn{4}{|l|}{ Maternal outcomes, $n$ (\%) } \\
\hline SLE flare during pregnancy & $25(33.3)$ & $4(40)$ & $14(32.6)$ \\
\hline Pre-eclampsia & $3(4.0)$ & $1(10)$ & $2(4.7)$ \\
\hline Eclampsia & $0(0)$ & $0(0)$ & $0(0)$ \\
\hline Gestational diabetes & $4(5.3)$ & $0(0)$ & $4(9.3)$ \\
\hline Venous thromboembolism & $1(1.3)$ & $1(10)$ & $0(0)$ \\
\hline Placenta praevia & $3(4)$ & $1(10)$ & $2(4.7)$ \\
\hline Post-partum haemorrhage & $1(1.3)$ & $0(0)$ & $0(0)$ \\
\hline Maternal death & $0(0)$ & $0(0)$ & $0(0)$ \\
\hline
\end{tabular}

SLE: systemic lupus erythematosus; APS: antiphospholipid syndrome; FGR: fetal growth restriction

${ }^{\mathrm{a}}$ Missing $=8,{ }^{\mathrm{b}}$ Missing $=6,{ }^{\mathrm{c}}$ Missing $=1,{ }^{\mathrm{d}}$ Missing=5

intrauterine fetal death, FGR, preterm delivery, fetal abnormality, congenital heart block or neonatal lupus occurred in half the pregnancies $(53.3 \%)$. The most common adverse fetal outcome was preterm delivery ( $<37$ weeks), which was $33.9 \%$ and this rate is similar to other cohort studies. ${ }^{21,22}$ Risk factors for preterm delivery in SLE patients include APS, lupus nephritis, arterial hypertension, high SLE activity prior to conception, SLE flares and glucocorticoid treatment during pregnancy. ${ }^{20,23-25}$ In the subgroup of SLE with APS patients, the rate of preterm delivery was higher $(60 \%)$. In addition, the majority (76\%) of our cohort were on glucocorticoids during pregnancy. A recent metaanalysis reported the pooled relative risk (RR) for preterm delivery in SLE patients versus controls was 2.05 (95\% CI 1.72-3.32). ${ }^{26}$ There were no predictive factors identified for all adverse fetal outcomes or adverse maternal outcomes in our cohort. In the subgroup analysis of preterm delivery, anti-Ro (SS-A) antibody positivity and hydroxychloroquine treatment were associated with a lower risk of preterm delivery. In the subgroup of anti-Ro (SS-A) antibody positive pregnancies, antimalarial usage, concomitant rheumatic diseases and SLE disease activity at baseline were comparable to the whole cohort. A large prospective study of anti-Ro positive women reported that the presence of anti-Ro antibodies was not associated with adverse pregnancy outcomes in both SLE patients and non-SLE patients. ${ }^{27}$ In a retrospective study, it was postulated that anti-Ro antibodies appear to have a favourable impact in patients with SLE, which is a severe systemic disease. Conversely they have been reported 
Table 3. Univariate mixed model analysis of the potential exploratory variables associated with preterm delivery

\begin{tabular}{|c|c|c|}
\hline & OR $(95 \%$ CI $)$ & $P$ value \\
\hline \multicolumn{3}{|l|}{ Race } \\
\hline Chinese & Reference & \\
\hline Malay & $4.2(0.9-19.6)$ & 0.070 \\
\hline Indian & - & 1.000 \\
\hline Others & - & 1.000 \\
\hline Smoker & $1.4(0.1-32.6)$ & 0.820 \\
\hline \multicolumn{3}{|l|}{ SLE clinical manifestations } \\
\hline Haematological & $1.8(0.3-11.4)$ & 0.540 \\
\hline Arthritis & $1.9(0.3-14.7)$ & 0.500 \\
\hline Renal & $0.9(0.3-4.9)$ & 0.920 \\
\hline Muco-cutaneous & $1.5(0.3-8.2)$ & 0.610 \\
\hline Serositis & - & 0.002 \\
\hline Neurological & $1.4(0.1-24.4)$ & 0.820 \\
\hline \multicolumn{3}{|c|}{ Immunological characteristics } \\
\hline anti-Ro (SS-A) & $0.4(0.4-0.4)$ & $<0.0001$ \\
\hline anti-La (SS-B) & $0.5(0.0-9.5)$ & 0.610 \\
\hline anti-cardiolipin IgG & $0.1(0.0-23.6)$ & 0.450 \\
\hline anti-cardiolipin IgM & $1.1(0.1-12.0)$ & 0.940 \\
\hline Lupus anticoagulant & $2.6(0.2-35.0)$ & 0.460 \\
\hline \multicolumn{3}{|l|}{ Medications } \\
\hline Prednisone & $4.8(0.4-64.4)$ & 0.230 \\
\hline Hydroxychloroquine & $0.0(0.0-0.1)$ & 0.008 \\
\hline Chloroquine & $2.2(0.1-43.0)$ & 0.600 \\
\hline Azathioprine & $3.3(0.5-23.5)$ & 0.240 \\
\hline Aspirin & $4.2(0.4-44.3)$ & 0.240 \\
\hline SLE with secondary APS & $3.7(0.3-39.8)$ & 0.290 \\
\hline SLE with lupus nephritis & $0.9(0.2-5.0)$ & 0.920 \\
\hline
\end{tabular}

OR: odds ratio; CI: confidence interval; aCL: anti-cardiolipin antibody; APS: antiphospholipid syndrome

to have an adverse effect in patients with milder autoimmune disease such as Sjogren's Syndrome. ${ }^{28}$

The rate of miscarriage was $15 / 75(20 \%)$ with the majority (13 pregnancies) being early miscarriages. There has been a reported reduction in the rates of fetal loss in SLE pregnancies from $43 \%$ in the period of $1960-1965$ to $17 \%$ in the period of $2000-2003$, reflecting improvement in disease management and pregnancy monitoring. ${ }^{29}$ Similarly, another literature review reported a comparable mean fetal loss rate of $19.5 \%$ in SLE women. ${ }^{30}$ This is in keeping with the rate of miscarriages in our cohort. Risk factors for pregnancy loss in SLE include APS, active SLE clinically and serologically (hypocomplementaemia and high anti-double stranded DNA antibodies). ${ }^{31}$ This was reflected in our subgroup of SLE with APS patients, which had a miscarriage rate of $40 \%(4 / 10)$.

The rate of caesarean section in our cohort was $57.1 \%$, which was higher than other cohorts. Prior cohorts of SLE pregnancies reported over a third of caesarean deliveries; $36.6 \%$ in a national US population-based study performed in $2000-2003^{5}$ and $32-39 \%$ in Northern European cohort of SLE pregnancies. ${ }^{20,32}$ This rate was also higher than the 2014 Singapore national caesarean rate in the general population of $37.4 \%{ }^{33}$ Indications for caesarean sections in SLE pregnancies are similar to the general population; however, the increased rates of complications such as pre-eclampsia or fetal distress occur more frequently in SLE pregnancies, leading to a higher rate of caesarean sections. ${ }^{34}$ Further research evaluating indications for caesarean section in these SLE pregnancies will be helpful to understand this trend, which is currently limited by the retrospective nature of our study.

The prevalence of pre-eclampsia was low in our cohort and occurred in 3 pregnancies (4\%). In contrast, the occurrence of pre-eclampsia in SLE patients is reported to be between $12 \%$ and $35 \%{ }^{8,23,35}$ The risk factors of 
pre-eclampsia include autoimmune diseases such as SLE, APS, nulliparity, previous pre-eclampsia, multifetal pregnancy and chronic hypertension. ${ }^{36,37}$ Of note, the majority of our patients $(88 \%)$ had quiescent SLE disease activity at baseline and more than half were multiparous. In addition, there were only 2 pregnancies with a prior history of pre-eclampsia, which may have contributed to the lower rate of pre-eclampsia in our cohort. Aspirin was prescribed in a third of our cohort (33.3\%). In the most recent recommendations for management of SLE patients, low-dose aspirin has been recommended for SLE women to prevent or delay the onset of gestational hypertension and pre-eclampsia. ${ }^{38,39}$ Similarly, the American College of Obstetricians and Gynaecologists, National Institute for Health and Care Excellence, and US Preventive Services Health Task Force recommend low-dose aspirin as prophylaxis in all patients at high risk of pre-eclampsia, which included patients with SLE or APS. ${ }^{40,41}$

SLE flares occurred in a third of our cohort. This finding is similar to those reported in previous studies with flare rates ranging between $19 \%$ and $68 \% .^{7-10}$ Predictors for flares identified included disease activity at time of conception, lupus nephritis and discontinuation of medications such as hydroxychloroquine. ${ }^{11,12}$ Hydroxychloroquine or chloroquine were prescribed in the majority of our cohort (64\%). In addition, the majority $(88 \%)$ had quiescent SLE disease activity at baseline. In keeping with previous reports, the most commonly affected organs in SLE flares were haematological $(44 \%)$, renal $(40 \%)$, mucocutaneous $(28 \%)$ and musculoskeletal $(16 \%))^{24,42,43}$ In our subgroup of SLE with lupus nephritis pregnancies, the SLE flare rate was similar.

Pregnancies in women with APS have an increased risk of adverse pregnancy outcomes including FGR, pre-maturity, pregnancy loss with recurrent early miscarriages or late miscarriages, pre-eclampsia and eclampsia. ${ }^{44}$ In our subgroup of SLE with secondary APS pregnancies, there was a higher rate of adverse fetal outcome $(8 / 10)$ and the most common was miscarriages. All SLE with APS pregnancies were treated with either aspirin or LMWH and 4 were treated with combination therapy. The recent guidelines recommend combination therapy with low-dose aspirin and prophylactic dose of LMWH in obstetric APS patients. ${ }^{38,39}$ In our cohort, all were obstetric APS except for 1 patient. There is conflicting data on pregnancy outcomes between pregnancies with obstetric versus thrombotic APS. Branham et al..$^{45}$ showed that patients with thrombotic APS had a higher incidence of adverse pregnancy outcomes whereas Hogden et al. ${ }^{46}$ demonstrated the converse with obstetric APS associated with a higher rate. The numbers in our subgroup of SLE with APS pregnancies were too small to evaluate this difference in pregnancy outcomes.

Our study has some limitations as this is a retrospective study with inherent biases including selection bias and missing information. On the other hand, our study reflects pregnancy outcomes of SLE patients in a real-life setting.

\section{Conclusion}

In our cohort, SLE pregnancies were associated with an increased adverse fetal and maternal outcome despite quiescent SLE disease activity at baseline. Caesarean section rate in SLE pregnancies was also higher than the national average rate. Anti-Ro (SS-A) antibody positivity and hydroxychloroquine treatment were associated with a lower risk of preterm delivery. SLE pregnancies with APS appeared to have a higher rate of adverse fetal outcomes and SLE flares despite therapy with aspirin or LMWH. Therefore, this emphasised the importance of preconception counselling, optimal timing of pregnancy, risk stratification and multidisciplinary management of SLE pregnancies to achieve optimal outcomes.

\section{REFERENCES}

1. Carter EE, Barr SG, Clarke AE. The global burden of SLE: prevalence, health disparities and socioeconomic impact. Nat Rev Rheumatol 2016;12:605-20.

2. Ekblom-Kullberg S, Kautiainen H, Alha P, et al. Reproductive health in women with systemic lupus erythematosus compared to population controls. Scand J Rheumatol 2009;38:375-80.

3. Clowse MEB, Chakravarty E, Costenbader KH, et al. Effects of infertility, pregnancy loss, and patient concerns on family size of women with rheumatoid arthritis and systemic lupus erythematosus. Arthritis Care Res (Hoboken) 2012;64:668-74.

4. Ostensen M, Clowse M. Pathogenesis of pregnancy complications in systemic lupus erythematosus. Curr Opin Rheumatol 2013; 25:591-596.

5. Clowse MEB, Jamison M, Myers E, et al. A national study of the complications of lupus in pregnancy. Am J Obstet Gynecol 2008; 199:127.e1-6.

6. Mehta B, Luo Y, Xu J, et al. Trends in maternal and fetal outcomes among pregnant women with systemic lupus erythematosus in the United States. Ann Intern Med 2019;171:164-71.

7. Cortés-Hernández J, Ordi-Ros J, Paredes F, et al. Clinical predictors of fetal and maternal outcome in systemic lupus erythematosus: a prospective study of 103 pregnancies. Rheumatology (Oxford) 2002;41:643-50.

8. Chakravarty EF, Colón I, Langen ES, et al. Factors that predict prematurity and preeclampsia in pregnancies that are complicated by systemic lupus erythematosus. Am J Obstet Gynecol 2005; 192:1897-904.

9. Smyth A, Oliveira GHM, Lahr BD, et al. A systematic review and meta-analysis of pregnancy outcomes in patients with systemic 
lupus erythematosus and lupus nephritis. Clin J Am Soc Nephrol 2010;5:2060-8.

10. Gladman DD, Tandon A, Ibañez D, et al. The effect of lupus nephritis on pregnancy outcome and fetal and maternal complications. J Rheumatol 2010;37:754-8.

11. Jara LJ, Medina G, Cruz-Dominguez P, et al. Risk factors of systemic lupus erythematosus flares during pregnancy. Immunol Res 2014;60:184-92.

12. Buyon JP, Kim MY, Guerra MM, et al. Predictors of pregnancy outcomes in patients with lupus: a cohort study. Ann Intern Med 2015; 163:153-163.

13. Hochberg MC. Updating the american college of rheumatology revised criteria for the classification of systemic lupus erythematosus. Arthritis Rheum 1997;40:1725.

14. Petri M, Kim MY, Kalunian KC, et al. Combined oral contraceptives in women with systemic lupus erythematosus. N Engl J Med 2005;353:2550-8.

15. Wilson $\mathrm{W}$ a, Gharavi AE, Koike $\mathrm{T}$, et al. International consensus statement on preliminary classification criteria for definite antiphospholipid syndrome. Arthritis Rheum 1999;42:1309-111.

16. Confidential Enquiry into Maternal and Child Health (CEMACH) : Perinatal Mortality 2007: United Kingdom. Available at: https://www. oaa-anaes.ac.uk/assets/ managed/editor/File/Report/2007 Perinatal mortality.pdf. Accessed on 14 September 2020.

17. Iliodromiti S, Mackay DF, Smith GCS, et al. Apgar score and the risk of cause-specific infant mortality: a population-based cohort study. Lancet 2014;384:1749-1755.

18. Pastore DEA, Costa ML, Surita FG. Systemic lupus erythematosus and pregnancy: the challenge of improving antenatal care and outcomes. Lupus 2019;28:1417-26.

19. Tedeschi SK, Guan H, Fine A, et al. Organ-specific systemic lupus erythematosus activity during pregnancy is associated with adverse pregnancy outcomes. Clin Rheumatol 2016;35:1725-32.

20. Jakobsen IM, Helmig RB, Stengaard-Pedersen K. Maternal and foetal outcomes in pregnant systemic lupus erythematosus patients: an incident cohort from a stable referral population followed during 1990-2010. Scand J Rheumatol 2015;44:377-84.

21. Wu J, Ma J, Bao C, et al. Pregnancy outcomes among chinese women with and without systemic lupus erythematosus: A retrospective cohort study. BMJ Open 2018;8:1-9.

22. Clark CA, Spitzer KA, Nadler JN, et al. Preterm deliveries in women with systemic lupus erythematosus. J Rheumatol 2003;30:2127-32.

23. Borella E, Lojacono A, Gatto M, et al. Predictors of maternal and fetal complications in SLE patients: a prospective study. Immunol Res 2014;60:170-6.

24. Saavedra MA, Cruz-Reyes C, Vera-Lastra O, et al. Impact of previous lupus nephritis on maternal and fetal outcomes during pregnancy. Clin Rheumatol 2012;31:813-9.

25. Chakravarty EF, Colón I, Langen ES, et al. Factors that predict prematurity and preeclampsia in pregnancies that are complicated by systemic lupus erythematosus. Am J Obstet Gynecol 2005; 192:1897-904.

26. Wei S, Lai K, Yang Z, et al. Systemic lupus erythematosus and risk of preterm birth: a systematic review and meta-analysis of observational studies. Lupus 2017;26:563-71.

27. Brucato A, Doria A, Frassi M, et al. Pregnancy outcome in 100 women with autoimmune diseases and anti-Ro/SSA antibodies: a prospective controlled study. Lupus 2002;11:716-21.

28. Mavragani CP, Dafni UG, Tzioufas AG, et al. Pregnancy outcome and anti-Ro/SSA in autoimmune diseases: a retrospective cohort study. Br J Rheumatol 1998;37:740-5.
29. Clark CA, Spitzer KA, Laskin CA. Decrease in pregnancy loss rates in patients with systemic lupus erythematosus over a 40-year period. J Rheumatol 2005;32:1709-12.

30. Yan Yuen S, Krizova A, Ouimet JM, et al. Pregnancy outcome in systemic lupus erythematosus (SLE) is improving: results from a case control study and literature review. Open Rheumatol J 2008; 2:89-98.

31. Clowse MEB, Magder LS, Petri M. The clinical utility of measuring complement and anti-dsDNA antibodies during pregnancy in patients with systemic lupus erythematosus. J Rheumatol 2011;38:1012-6.

32. Wallenius M, Salvesen K, Daltveit AK, et al. Systemic lupus erythematosus and outcomes in first and subsequent births based on data from a national birth registry. Arthritis Care Res 2014; 66:1718-24.

33. Chi C, Pang D, Aris IM, et al. Trends and predictors of cesarean birth in Singapore, 2005-2014: a population-based cohort study. Birth 2018:45:399-408.

34. Fischer-Betz R, Specker C. Pregnancy in systemic lupus erythematosus and antiphospholipid syndrome. Best Pract Res Clin Rheumatol 2017;31:397-414.

35. Arkema E V, Palmsten K, Sjöwall C, et al. What to expect when expecting with systemic lupus erythematosus (SLE): a populationbased study of maternal and fetal outcomes in SLE and pre-SLE. Arthritis Care Res (Hoboken) 2016;68:988-94.

36. Duckitt K, Harrington D. Risk factors for pre-eclampsia at antenatal booking: systematic review of controlled studies. BMJ 2005;330:565.

37. Bartsch E, Medcalf KE, Park AL, et al. Clinical risk factors for pre-eclampsia determined in early pregnancy: systematic review and meta-analysis of large cohort studies. BMJ 2016; 353:i1753

38. Andreoli L, Bertsias GK, Agmon-Levin N, et al. EULAR recommendations for women's health and the management of family planning, assisted reproduction, pregnancy and menopause in patients with systemic lupus erythematosus and/or antiphospholipid syndrome. Ann Rheum Dis 2017;76:476-85.

39. Sammaritano LR, Bermas BL, Chakravarty EE, et al. 2020 American college of rheumatology guideline for the management of reproductive health in rheumatic and musculoskeletal diseases. Arthritis Rheumatol 2020;72:529-56.

40. LeFevre ML. Low-dose aspirin use for the prevention of morbidity and mortality from preeclampsia: U.S. Preventive Services Task Force recommendation statement. Ann Intern Med 2014;161:819-26.

41. ACOG Committee Opinion No. 743: Low-Dose Aspirin Use During Pregnancy. Obstet Gynecol 2018;132:e44-52.

42. Imbasciati E, Tincani A, Gregorini G, et al. Pregnancy in women with pre-existing lupus nephritis: predictors of fetal and maternal outcome. Nephrol Dial Transplant 2009;24:519-25.

43. Clowse MEB. Lupus activity in pregnancy. Rheum Dis Clin North Am 2007;33:237-52.

44. Schreiber K, Sciascia S, de Groot PG, et al. Antiphospholipid syndrome. Nat Rev Dis Primers 2018;4:18005.

45. Bramham K, Hunt BJ, Germain S, et al. Pregnancy outcome in different clinical phenotypes of antiphospholipid syndrome. Lupus 2010;19:58-64.

46. Hogden A, Antovic A, Berg E, et al. Obstetric outcomes in patients with primary thrombotic and obstetric antiphospholipid syndrome and its relation to the antiphospholipid antibody profile. Lupus 2019;28:868-77. 